\title{
Weitergabe einer perioperativen Antibiotikaprophylaxe
}

\author{
C. Lanckohr
}

\section{Einleitung zur Rubrik}

In dieser Rubrik werden Experten, die die aktuelle Literatur sehr gut überblicken und umfassende klinische Erfahrung haben, Antworten auf Ihre Fragen liefern, die sich nicht so einfach aus der Literatur beantworten lassen. Wenn Sie also eine außergewöhnliche Frage haben, auf die Sie sich schon lange eine Antwort wünschen, freuen wir uns auf Ihre Zuschrift an Gabi.Hasenmaile@thieme.de.

\section{Ask the Expert}

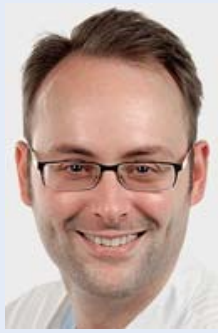

C. Lanckohr
Die Frage, wie lange eine perioperative Antibiotikaprophylaxe auf der Intensivstation weiter gegeben werden sollte, wird kontrovers diskutiert. Gibt es Leitlinien oder klare Handlungsanweisungen, wann und gegebenenfalls wie lange eine perioperative Antibiotikaprophylaxe weiter geführt werden sollte?

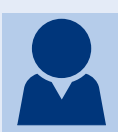

Die perioperative Antibiotikaprophylaxe (PAP) ist eine zentrale Maßnahme zur Verhütung der postoperativen Wundinfektion, die immer noch die häufigste nosokomiale Infektion in Deutschland darstellt [1]. Eine Reihe von Teilaspekten müssen berücksichtigt werden, um die PAP optimal durchzuführen [2]. Im Vordergrund stehen hier insbesondere die zeitgerechte Applikation ca. 15-60 Minuten vor Hautinzision, eine adäquate Substanzwahl zur Abdeckung der ortsständigen Flora sowie die regelmäßige Repetition entsprechend der Halbwertszeiten der verwendeten Antibiotika.

Ein im klinischen Alltag oft kontrovers diskutiertes Thema ist die Frage nach der Dauer der PAP. In vielen operativen Kliniken scheint eine Fortsetzung der Antibiotika im Sinne einer „extendierten Prophylaxe“ typische Praxis zu sein, wie nationale Punkt-PrävalenzUntersuchungen suggerieren [1]. Dieses Verhalten ist hochproblematisch und bedarf einer vermehrten Aufmerksamkeit.
Es existieren keine belastbaren wissenschaftlichen Erkenntnisse dafür, dass eine Fortsetzung der prophylaktisch verabreichten Antibiotika nach Ende der Operation sinnvoll sein könnte [2]. Eine Reduktion der Wundinfektionsrate kann hierdurch offenbar nicht erreicht werden. Als einzige Ausnahme gelten derzeit herzchirurgische Eingriffe mit Einsatz der Herz-Lungen-Maschine, wo eine Ausweitung der PAP auf einen Zeitraum von ca. 24 Stunden Vorteile bringen könnte [3]. Klar abzugrenzen von der Prophylaxe sind klinische Situationen, in denen ein operativer Eingriff zur Behandlung einer Infektion durchgeführt wird. Hier ergibt sich die Indikation zur Antibiotikatherapie aus der Infektion, und eine postoperative Fortsetzung der Antiinfektiva ist als Therapie zu bewerten, nicht als extendierte Prophylaxe.

Aus diesen Überlegungen folgt die Erkenntnis, dass für den weit überwiegenden Teil aller Eingriffe eine Fortsetzung der Antibiotikagabe nach Ende der Operation nicht indiziert ist. Diese leider noch oft anzutreffende klinische Praxis ist ein wichtiger Ansatzpunkt, um die Menge der verordneten Antibiotika zu reduzieren [1]. Bei fehlendem klinischem Nutzen wird durch eine sinnlos verlängerte PAP ein vermeidbarer Resistenzdruck erzeugt, den es dringend zu reduzieren gilt. Entsprechende Empfehlungen können in internationalen Leitlinien detailliert nachgelesen werden [2].

Interessenkonflikt: Der Autor gibt an, dass kein Interessenkonflikt besteht.

\section{Literatur}

1 Behnke M, Hansen S, Leistner R et al. Nosocomial infection and antibiotic use: a second national prevalence study in Germany. Dtsch Arztebl Int 2013; 110: 627-633

2 Bratzler DW, Dellinger EP, Olsen KM et al. Clinical practice guidelines for antimicrobial prophylaxis in surgery. Am J Health Syst Pharm 2013; 70: 195-283

3 Kappeler R, Gillham M, Brown NM. Antibiotic prophylaxis for cardiac surgery. J Antimicrob Chemother 2012; 67: 521 - 522 